Recibido: 1 diciembre 2020 | Aceptado: 15 diciembre 2020 | Publicado: 23 diciembre 2020

Cita: Camargo, L. y Cortés, R. (2020). Desarrollo y mejora de la competencia pragmática en niños y adolescentes con trastorno del espectro autista y lenguaje funcional. Normas, 10(1). doi: http://dx.doi.org/10.7203/Normas.v10i1.19279

\title{
DESARROLLO Y MEJORA DE LA COMPETENCIA PRAGMÁTICA EN NIÑOS Y ADOLESCENTES CON TRASTORNO DEL ESPECTRO AUTISTA Y LENGUAJE FUNCIONAL
}

\author{
DEVELOPMENT AND IMPROVEMENT OF THE PRAGMATIC COMPETENCE IN \\ CHILDREN AND ADOLESCENTS WITH AUTISTIC SPECTRUM DISORDER AND \\ FUNCTIONAL LANGUAGE
}

\section{Laura Camargo Fernández}

\section{Regina Cortés García}

Universitat de les Illes Balears

Resumen

Entre los aspectos de la conducta social alterados en las personas con trastorno del espectro autista y lenguaje funcional (TEA grado 1, dentro del que se incluye el síndrome de Asperger) la competencia pragmática es uno de los más estudiados. No obstante, faltan trabajos empíricos sobre la posible mejora de los aspectos comunicativos problemáticos con el desarrollo evolutivo y la praxis comunicativa. El objetivo de esta investigación es revisar los déficits pragmáticos más habituales en niños y adolescentes con TEA y lenguaje funcional y comprobar si la práctica del uso social del lenguaje puede implicar una mejora en su competencia pragmática. Para llevar a cabo esta investigación, se ha realizado una recogida de datos a través de un cuestionario de 26 preguntas entre 110 padres y madres de niños y jóvenes de edades comprendidas entre los 6 y los 18 años diagnosticados con TEA grado 1. Los resultados confirman, en línea con trabajos anteriores, cuáles son los aspectos de la competencia pragmática más problemáticos en estos niños y adolescentes -asociados a la comprensión y expresión de la intención comunicativa, la dinámica conversacional, la comunicación no verbal y la comprensión del lenguaje figurado-, a la par que revelan que algunas de las habilidades alteradas se pueden adquirir o mejorar con el paso de los años. El estudio confirma, asimismo, el hecho de que la adquisición o mejora de ciertas destrezas no garantiza que puedan ponerse en práctica en todos los contextos, dadas las dificultades para generalizar aprendizajes que presentan las personas con TEA.

PALABRAS CLAVE: competencia pragmática, trastorno del espectro autista, síndrome de Asperger, análisis de la conversación, comunicación no verbal.
Abstract

Among the aspects of altered social behavior in people with autism spectrum disorder and functional language (ASD grade 1, which includes Asperger syndrome), pragmatic competence is one of the most studied. However, there is a lack of empirical work on the possible improvement of problematic communicative aspects in relation with evolutionary development and communicative praxis. The objective of this research is to review the most common pragmatic deficits in children and adolescents with ASD and functional language and to verify whether the practice of social use of language can imply an improvement in their pragmatic competence. To carry out this research, data was collected through a questionnaire of 26 questions among 110 parents of children and young people aged between 6 and 18 years diagnosed with ASD grade 1. The results confirm, in line with previous works, which are the most problematic aspects of pragmatic competence in these children and adolescents -associated with the understanding and expression of communicative intention, conversational dynamics, non-verbal communication and the understanding of figurative language-, at the same time that they reveal that some of the altered skills can be acquired or improved over the years. The study also confirms the fact that the acquisition or improvement of certain skills does not guarantee that they can be put into practice in all contexts, given the difficulties in generalizing learning that people with ASD present.

KEY WORDS: pragmatic competence, autism spectrum disorder, Asperger syndrome, conversation analysis, non-verbal communication. 


\section{INTRODUCCIÓN}

Una de las principales características del trastorno del espectro autista (TEA) es el déficit persistente en la capacidad de iniciar y sostener la interacción social recíproca y en la comunicación interpersonal. Los individuos con TEA y lenguaje funcional (TEA grado 1, que incluye el síndrome de Asperger ${ }^{1}$, SA), cuya conducta comunicativa es objeto de estudio en esta investigación, muestran habilidades no problemáticas en cuanto al lenguaje verbal, incluyendo un desarrollo normal de la competencia semántica, a la par que exhiben aspectos claramente alterados en la competencia pragmática. Una característica sorprendente e incuestionada del perfil lingüístico de las personas con TEA y lenguaje funcional es que si bien muestran una inteligencia normal y un conocimiento adecuado de las normas gramaticales, y en ocasiones un amplio vocabulario y capacidad lingüística formal avanzada con respecto a niños de su misma edad, presentan problemas severos de comunicación cuya evidencia más clara se encuentra en los problemas de reconocimiento de la intención comunicativa, la dinámica conversacional, la comunicación no verbal y la comprensión del lenguaje figurado (Martín Borreguero 2005, Tordera Yllescas 2007, Reboul et alii 2012, Rodríguez Muñoz 2013, Barthélémy et alii 2019).

Las personas diagnosticadas con Asperger o TEA grado 1 muestran dificultades para relacionarse socialmente, especialmente con sus iguales, además de intereses restringidos, alteración de la comunicación no verbal e inflexibilidad cognitiva y de comportamiento. También se caracterizan por presentar dificultades a la hora de abstraer conceptos y por presentar una coherencia central débil frente a un mejor procesamiento de los detalles (Happé 1999) ${ }^{2}$. Los individuos con este diagnóstico interpretan el lenguaje de forma literal y muestran dificultades tanto en las funciones ejecutivas y de planificación como a la hora de interpretar sus sentimientos y los de los demás. Asimismo, las personas con TEA exhiben problemas de diversos grados en relación con la cognición social y la teoría de la mente, es decir, dificultades en aspectos generales sociales y cognitivos de la representación del mundo en las mentes, como la capacidad de adjudicar estados mentales a los demás (Baron-Cohen 2001, Flavell 2004, Tordera Yllescas 2007), hecho también relacionado con la conciencia metapragmática y que

\footnotetext{
1 En la revisión de criterios diagnósticos publicada por la American Psychiatric Association en 2013, el DSM 5 (Diagnostic and Statistic Manual of Mental Disorders 5), el síndrome de Asperger pasó a englobarse en la categoría general de "Trastorno del Espectro del Autismo" (TEA) y se eliminó como categoría independiente. En el CIE 10 de 1992 (Clasificación Internacional de Enfermedades de la Organización Mundial de la Salud 10), el síndrome de Asperger se mantiene como entidad propia, pero esta desaparecerá y pasará a formar parte del TEA cuando entre en vigor el próximo CIE 11 en enero de 2022. Aunque entre muchas asociaciones de familiares se mantiene la denominación social de síndrome de Asperger por la identidad generada en torno a ese nombre entre individuos así diagnosticados y sus familias, generalmente hemos elegido para este trabajo la denominación TEA y lenguaje funcional o TEA grado 1, que es el diagnóstico que reciben hoy y recibirán en el futuro las personas con Asperger. No obstante, mantendremos esta última denominación siempre que hagamos referencia a investigaciones centradas en dicho síndrome que se hayan publicado con anterioridad a las citadas revisiones.

2 De acuerdo con Happé (1999: 217), «they show detail-focused processing in which features are perceived and retained at the expense of global configuration and contextualised meaning». Aunque hay autores que niegan o matizan la hipótesis de la coherencia central débil, los problemas de contextualización son -como se demuestra también en este estudio- habituales en las personas con TEA.
}

Normas (ISSN: 2174-7245) | 
Desarrollo y mejora de la competencia pragmática en niños y adolescentes con trastorno del espectro autista y lenguaje funcional | L. Camargo y R. Cortés

se observa en los problemas para interpretar, entre otros aspectos del lenguaje no literal, las implicaturas irónicas (Camargo Fernández 2009, Pexman et alii 2011).

En suma, las personas con TEA grado 1 pueden hablar con fluidez y construir oraciones gramaticalmente correctas, lo cual no les basta para asegurarse el éxito en sus procesos comunicativos. Otra tarea que entraña una gran dificultad para los afectados es la de «aplicar las reglas del lenguaje socialmente contextualizado e imponer límites significativos en su capacidad para responder de acuerdo con las expectativas sociales de la cultura de su entorno» (Martín-Borreguero 2005: 118).

La bibliografía reciente muestra numerosísimas investigaciones sobre TEA que han puesto el foco en todas las habilidades comunicativas afectadas en niños durante las primeras etapas del aprendizaje lingüístico y la socialización. Pero como demostraron con claridad en su pormenorizada revisión Parsons et alii (2017), escasean los trabajos sobre el desarrollo y posibilidad de mejora de las habilidades comunicativas en adolescentes y jóvenes con TEA 3 . Partiendo de la hipótesis de que los aspectos de la competencia pragmática alterados en este tipo de TEA son susceptibles de mejora, tres son los objetivos de este trabajo4:

1) Revisar los déficits pragmáticos en niños y jóvenes con TEA grado 1.

2) Comprobar cuáles son los aspectos de la comunicación interpersonal que entrañan mayor complejidad para los niños y jóvenes con TEA grado 1 y si pueden mejorar con el desarrollo y la práctica comunicativa.

3) Confirmar si un individuo con este diagnóstico puede adquirir las habilidades pragmáticas necesarias para una comunicación exitosa y si, a pesar de su dificultad para generalizar aprendizajes (Maristany 2002: 7), puede llegar a ponerlas en práctica con interlocutores desconocidos o en situaciones nuevas.

Para llevar a cabo esta investigación, se ha realizado una encuesta entre 110 familias de niños y jóvenes con TEA y lenguaje funcional diagnosticados bien como síndrome de Asperger o bien como TEA grado 1, con edades comprendidas entre los 6 y los 18 años. El cuestionario se ha repartido entre asociaciones de familias con autismo, discapacidad e inclusión y entre grupos de padres de niños con TEA tanto de Baleares como del resto de España ${ }^{5}$.

3 Martín-Borreguero (2005: 121) considera que el conocimiento de las competencias lingüísticas y déficits pragmáticos de las personas con este diagnóstico permitiría llevar a cabo una evaluación y posterior intervención para facilitar su progreso en la comprensión del mundo social.

4 Este artículo está inspirado en el trabajo de fin de grado de Regina Cortés Rodríguez, realizado bajo la tutorización de Laura Camargo Fernández, y presentado en la Universitat de les Illes Balears bajo el título Autismo con lenguaje funcional: un enfoque pragmático: http://hdl.handle.net/11201/153808.

5 Para la elaboración del cuestionario hemos contado con el asesoramiento de Marta Cruz, formadora en TIC a colectivos en riesgo de exclusión social e impulsora de una plataforma que agrupa a más de 3.000 familias de niños con TEA, además de Presidenta del "Grup d'Ajuda Mútua Inclusiva" (GAMIN), a quien agradecemos sinceramente su ayuda e implicación. Ha sido,

Normas (ISSN: 2174-7245)| 
Desarrollo y mejora de la competencia pragmática en niños y adolescentes con trastorno del espectro autista y lenguaje funcional | L. Camargo y R. Cortés

\section{MARCO TEÓRICO. ASPECTOS PRAGMÁTICOS ALTERADOS EN PERSONAS CON TEA Y LENGUAJE FUNCIONAL}

La revisión de las dificultades comunicativas en niños y jóvenes con SA o TEA grado 1 parte de los trabajos de Martín-Borreguero (2005) y de Rodríguez Muñoz (2012).

La primera autora toma como base el modelo de Twachtman-Cullen (1998), quien considera que la competencia pragmática de las personas evoluciona con los años y depende de la adquisición de numerosos conocimientos y habilidades sociales, por lo que es esperable que a lo largo de la vida los individuos puedan desarrollar y mejorar sus habilidades comunicativas. Destaca también que los individuos deben adquirir los conocimientos adecuados y la comprensión social para poder emitir juicios correctos y percibir las necesidades y sentimientos de las personas con las que entablan una conversación, además de ser capaces de comprender y aplicar las reglas del discurso para lograr una comunicación exitosa. Contempla dentro de la competencia pragmática la habilidad necesaria para usar y comprender los aspectos quinésicos y paralingüísticos de la comunicación.

A partir de estas premisas, Martín-Borreguero (2005: 118) expone cuáles son las habilidades y déficits pragmáticos asociados al SA. Entre los segundos, destaca el interés de los afectados por iniciar la interacción social, a la par que la ausencia de reciprocidad en el discurso social o la conversación, mientras que dentro de las habilidades incluye una comunicación adecuada de las intenciones simples, si bien señala que los afectados presentan dificultades a la hora de transmitir intenciones más complejas. Pese a la fluidez verbal y el interés por transmitir información que los afectados por SA presentan, la autora reconoce también un déficit en la capacidad de detectar las necesidades del interlocutor y problemas para cambiar de tópico conversacional. Finalmente, concluye que las personas con SA tienen una gran capacidad para retener información, pero muestran dificultades para comprender las pautas no verbales de comunicación.

Por su parte, Rodríguez Muñoz (2012) evalúa la competencia y la conciencia pragmática de las personas SA a partir del estudio del comportamiento comunicativo de adultos con este síndrome y compara su competencia pragmática con la de adultos neurotípicos. Los resultados del estudio concluyen que los adultos diagnosticados no muestran, por regla general, diferencias relevantes en los ítems referidos al área textual (mensaje, dominios léxico y gramatical), pero sí en las áreas enunciativa e interactiva, en las cuales exhibieron una conciencia pragmática deficitaria en comparación con los adultos neurotípicos.

A partir de los hallazgos de estas investigaciones, así como de otras citadas en el apartado anterior, hemos agrupado los déficits pragmáticos característicos del TEA grado 1 o SA en

asimismo, imprescindible para este estudio la participación de 110 familias integradas en estos colectivos a las cuales queremos también agradecer su colaboración respondiendo al cuestionario que dio base al citado trabajo de fin de grado. 
Desarrollo y mejora de la competencia pragmática en niños y adolescentes con trastorno del espectro autista y lenguaje funcional | L. Camargo y R. Cortés

cuatro ejes: a) la expresión y el reconocimiento de la intención comunicativa del hablante; b) la dinámica conversacional; c) la comunicación no verbal; y d) la comprensión del lenguaje no literal.

\subsection{La intención comunicativa}

El reconocimiento de la intención de su mensaje por parte del destinatario no parece ser un objetivo de la comunicación en las personas con TEA grado 1, dado que se comunican esencialmente para satisfacer sus necesidades emocionales y físicas. A consecuencia de sus problemas de contextualización, presentan dificultades a la hora de reconocer las implicaturas conversacionales particularizadas (Rodríguez Muñoz 2019). En cambio, en cuanto a las implicaturas conversacionales generalizadas (IGC), hay dos tipos de línea: la de los investigadores que muestran que los problemas pragmáticos de las personas con TEA no se manifiestan en todo el componente pragmático y no afectan a las implicaturas escalares, como Schaeken et alii (2018) y las de los que los que demuestran que los niños con TEA manifiestan limitaciones en la comprensión de las IGC de forma general (Pastor-Cerezuela et alii 2018).

En lo que hay consenso entre los especialistas es en que la conversación de estas personas suele versar sobre sus temas de interés, hasta el punto de que da la impresión de que no necesitan del interlocutor, presentando una mayor tendencia al monólogo que al diálogo. La comunicación, por tanto, parece responder más a la necesidad de hablar de sus intereses restringidos que a provocar algún tipo de reacción en el destinatario. Por otra parte, se constata un claro déficit a la hora de comprender las intenciones comunicativas de los demás, por lo que a menudo responde de manera inapropiada en la interacción comunicativa cara a cara. Como señala Martín-Borreguero (2005: 119):

\footnotetext{
La persona con el SA tiende a comunicar con prontitud sus preferencias, sus deseos de obtener o conocer algo en concreto, sus aversiones y sus intereses. Sin embargo, la emisión de comentarios de naturaleza social acerca de una actividad llevada a cabo por otra persona, la iniciación de una conversación social acerca de eventos diarios y rutinarios, la demostración de interés y deseo por averiguar los gustos de otra persona son actos significativamente menos frecuentes.
}

\subsection{La dinámica conversacional}

Las personas afectadas con TEA grado 1 tienen dificultades para saber cuándo intervenir en las conversaciones y es frecuente que no entiendan las señales para participar en el baile conversacional ni para atender a los mecanismos de alternancia de turnos, manifestando problemas para seguir las normas estratégicas de la conversación: saber escuchar, respetar la continuidad entre unidades, tanto estructurales como temáticas y demostrar, además, que se está escuchando; saber tomar la palabra y saber aceptar la interrupción e interrumpir mediante señales verbales o no verbales (mirada y gestos); saber mantener la palabra, a base de repeticiones breves o de pausas oralizadas, o saber cederla mediante pausas, vocativos, preguntas o silencios, entre otros aspectos destacados de la dinámica conversacional (Gallardo 1996, Cestero 2000, Camargo y Méndez 2014).

Normas (ISSN: 2174-7245) | 
Desarrollo y mejora de la competencia pragmática en niños y adolescentes con trastorno del espectro autista y lenguaje

funcional | L. Camargo y R. Cortés

De acuerdo con Martos Pérez (2007: 10-11), la discapacidad social que caracteriza a las personas con síndrome de Asperger o TEA grado 1 se refleja en los aspectos emocionales que intervienen en la comunicación, como el reconocimiento de las propias emociones y de las de los interlocutores o el manejo adecuado de las emociones en función del contexto; en la reciprocidad en la comunicación, en el diálogo, en el dominio del turno de palabra, en el uso correcto de la mirada como demarcación de los turnos y en la identificación del cambio de rol de emisor a receptor. También presentan dificultades para distinguir entre la información que ya se ha proporcionado y la nueva, a la hora de hacer las aclaraciones que puedan precisarse durante la conversación. Finalmente, carecen de los recursos adecuados para asegurarse de que el oyente comprende lo que se le está explicando, y la ya mencionada obsesión por determinadas temáticas se traduce en dificultades para reconocer las normas que indican cuándo y cómo cambiar de tema. El individuo Asperger, de hecho, puede resultar «abrumador en su discurso si se tratan temas de su interés. No tiene medida» (Maristany 2002: 13).

\subsection{La comunicación no verbal}

La comunicación no verbal juega un papel muy destacado en la comunicación eficaz: miradas o gestos suelen bastar para interpretar un cambio de turno de palabra, cambiar de tema, comprender la intención del emisor o incluso poner fin a la comunicación si se perciben gestos de aburrimiento o cansancio en el interlocutor (Cestero 2014). En el caso del individuo con TEA, los elementos no lingüísticos de la comunicación también se muestran significativamente alterados.

Martín-Borreguero (2005: 119) explica al respecto que en el ámbito de la expresión no verbal destacan "los déficits en la modulación de los turnos a través del contacto ocular, en la expresión flexible y coordinada de gestos manuales y corporales, en la exhibición de expresiones faciales congruentes con el contenido de la conversación", además de en "la adopción de posturas corporales correctas, en la prosodia de la voz y en el ritmo de habla". En referencia a la comprensión de las pautas no verbales de comunicación, la autora señala que datos clínicos han revelado que los afectados presentan problemas a la hora de interpretar los gestos faciales de los otros, así como a la de vincular estas expresiones al tono de voz, por lo que puede afirmarse que la prosodia es otro de los aspectos paralingüísticos que se ve afectado en los individuos con autismo y lenguaje funcional.

\subsection{La comprensión del lenguaje figurado y las unidades fraseológicas}

El reconocimiento del lenguaje figurado, como el que aparece en las metáforas o en las unidades fraseológicas (UFS) o la ironía es otro de los aspectos problemáticos para las personas diagnosticadas con TEA grado 1 o SA.

Normas (ISSN: 2174-7245) | 
Desarrollo y mejora de la competencia pragmática en niños y adolescentes con trastorno del espectro autista y lenguaje funcional | L. Camargo y R. Cortés

La interpretación de las metáforas se basa en la existencia de patrones de inferencia que funcionan de manera regular y en los que se pueden observar tres tipos de estrategias: de reconocimiento, para determinar si hay que buscar una interpretación metafórica; de cálculo de los valores que se pueden asignar a la entidad con respecto a la cual dos elementos son semejantes y de restricción, esto es, estrategias para restringir las posibilidades calculadas, e identificar el factor concreto que sirve como fundamento de la metáfora (Searle 1993). Tal como se recoge en la revisión exhaustiva y reciente de Kalandadze et alii (2019), los individuos con TEA presentan mayores dificultades para el reconocimiento del significado de las metáforas en relación también con las propiedades del tipo de metáfora de que se trate. En cuanto a las UFS, al tratarse de enunciados automatizados que no se interpretan a través de la suma de los significados literales de sus unidades léxicas (Timofeeva y Ruiz Gurillo 2018) provocan igualmente problemas de interpretación.

La persona que padece el tipo de autismo grado 1 suele entender el lenguaje ad pedem litterae, por lo que metáforas, unidades fraseológicas, así como otras formas que requieren trabajo inferencial y teoría de la mente, como el humor y la ironía o el sarcasmo suelen ser interpretados de manera literal (Pexman et alii 2011, Rodríguez Muñoz 2013). Así lo explica también Martos Pérez (2007: 13):

\footnotetext{
Con los actos de habla indirectos comunicamos más de lo que decimos literalmente, requiriéndose, por tanto, de conocimiento compartido para su comprensión (se precisa un uso adecuado, efectivo y rápido de habilidades de teoría de la mente). Ejemplos prototípicos de actos de habla indirectos son las ironías, las metáforas, las mentiras, las bromas, etc. La característica comprensión literal que presentan las personas con síndrome de Asperger, hacen que manifiesten claros y patentes signos de discapacidad en la comprensión y uso de los actos de habla indirectos. Con mucha frecuencia este particular tipo de discapacidad puede dar lugar a actos de mofa y burla a los que incomprensiblemente y sin las armas y herramientas necesarias tienen que enfrentarse las personas con síndrome de Asperger.
}

\section{METODOLOGÍA}

A partir de la constatación de los principales aspectos de la competencia pragmática alterados en las personas con TEA grado 1 y con el fin de comprobar en qué medida dichos aspectos eran susceptibles de mejora con el desarrollo evolutivo y la praxis comunicativa, se realizó una encuesta entre progenitores de niños y jóvenes diagnosticados con TEA grado 1 o SA en la que participaron 110 familias con hijos de edades comprendidas entre los 6 y los 18 años.

Basándonos en los campos problemáticos de la competencia pragmática ya descritos, elaboramos el siguiente cuestionario y lo distribuimos entre las familias participantes a través de Google Forms:

1. ¿A qué grupo de edad pertenece su hijo/a? (6- 14. ¿Hace movimientos repetitivos o inusuales $8,9-12,13+)$ mientras conversa con otra persona?

Normas (ISSN: 2174-7245) 
Desarrollo y mejora de la competencia pragmática en niños y adolescentes con trastorno del espectro autista y lenguaje funcional | L. Camargo y R. Cortés

2. Indique si su hijo/a ha recibido o no terapia. En caso afirmativo especifique la terapia o terapias recibidas

3. Cuando su hijo/a ve a personas hablando, ¿sabe cuándo intervenir en la conversación?

15. ¿Utiliza una entonación adecuada al hablar?

16. ¿Utiliza un vocabulario adecuado a su edad y al contexto? No es adecuado, por ejemplo, usar palabras complejas para su edad.

4. Durante una conversación, ¿sabe respetar el turno de palabra?

17. ¿Su hijo/a entiende frases del tipo "ponerse las pilas" o "estar en las nubes"?

5. En el caso de que su hijo sepa respetar el turno de palabra, ¿cree que lo respetará si el tema sobre

18. ¿Se dirige de la misma forma a un profesor el que están hablando es de su interés?

\section{6. ¿Presta atención en una conversación?} que a un niño?

7. ¿Responde a lo que se le pregunta?

19. Cuando le gusta mucho un tema, ¿habla de él sin parar?

20. Cuando habla de sus temas, ¿se da cuenta de si los demás le escuchan con atención o se aburren?

8. ¿Cuándo se dirigen a él o ella mira a la persona que le habla?

21. En el caso de que su hijo sepa reconocer que la persona con la que habla se aburre, ¿cree que dejaría de hablar de un tema aunque este le apasione?

9. ¿Mantiene la mirada durante la conversación?

22. ¿Cambia de tema de forma abrupta, pasando a un contenido que no tiene nada que ver con la conversación que se estaba manteniendo?

10. En el caso de que mantenga la mirada durante una conversación, ¿cree que la mantendría igualmente con personas desconocidas o en un entorno con el que no está familiarizado?

11. ¿Entiende su hijo/a los gestos que se hacen durante una conversación (guiñar el ojo, resoplar, gestos con las manos...)?

23. ¿Puede mantener una conversación fluida por teléfono?

12. $\mathrm{Si}$ entiende los gestos durante una conversación, ¿cree que podrá captarlos si habla con personas que no conoce o en un ambiente que no le resulta familiar?

24. ¿Tiene dificultades para aceptar otros puntos de vista?

25. En el caso de que su hijo/a entienda actualmente bromas, ¿cree que sería capaz de distinguir una broma que no ha escuchado nunca? 


\author{
13. ¿Mantiene la distancia interpersonal durante \\ una conversación (no se aleja ni se acerca \\ demasiado)? \\ 26. ¿Cree que los estímulos externos (ambiente, \\ ruido, luces...) pueden condicionar la atención y \\ respuestas de su hijo durante una conversación? \\ Cuadro I. Cuestionario sobre competencia pragmática en niños y adolescentes con TEA grado
}

Como se observa, todas las preguntas tienen relación con los campos que hemos desglosado en el punto anterior excepto la última, con la que se pretende analizar la relevancia de los estímulos externos en una conversación teniendo en cuenta que "en el SA se aprecian numerosos síntomas relacionados con hiperreactividad o hiporreactividad sensorial" (Artigas 2000: 37).

En todo el cuestionario se ofreció a las familias participantes la posibilidad de contestar 'sí' o 'no' y en muchas de ellas se dio la opción de precisar si sus hijos adquirieron los aprendizajes al mismo tiempo que sus coetáneos o si lo hicieron posteriormente, para comprobar así si niños y jóvenes con autismo y lenguaje funcional tienen la misma evolución en lo que a competencia pragmática se refiere.

En preguntas cuya contestación afirmativa supone no haber superado un hito, también se dio la posibilidad de contestar "no, lo hacía, pero ha dejado de hacerlo". Es el caso, por ejemplo, de la cuestión relativa a las estereotipias motoras (movimientos repetitivos); en este punto la citada opción aporta mucha información porque ayuda a discernir si el niño no ha presentado estereotipias o si ha podido corregirlas a lo largo de su desarrollo.

Varias preguntas se introdujeron con la finalidad de evaluar si el aprendizaje adquirido por los niños y jóvenes es válido en cualquier contexto, esto es, si podrán aplicar el conocimiento aprendido cuando cambie el contexto de una conversación, los interlocutores, el tema o el ambiente, mostrando así la misma competencia pragmática y adaptación contextual de un individuo neurotípico. De esta forma, y partiendo de su experiencia y expectativas, los progenitores podían responder no solo si su hijo respetaría el turno de palabra, sino también si seguiría respetándolo cuando el tema fuera de su interés. Igualmente, se les planteó si mantendría la mirada con desconocidos, entendería los gestos en un ambiente que no le fuese familiar, distinguiría bromas nuevas o dejaría de hablar de un tema que le apasione si detecta que el interlocutor se aburre.

La opción 'a veces' se ha evitado en el cuestionario para lograr la máxima objetividad posible en las respuestas. En este caso, tuvimos en cuenta que quienes responderían a las preguntas planteadas eran padres y madres de niños con un trastorno de características comunicativas y de socialización complejas y sin características físicas evidentes; de hecho, en los casos en los que estos niños presentan un desarrollo normal del lenguaje, es frecuente que se tarde años en dar con el diagnóstico, por lo que muchos padres se han encontrado, de forma repentina 
Desarrollo y mejora de la competencia pragmática en niños y adolescentes con trastorno del espectro autista y lenguaje funcional | L. Camargo y R. Cortés

tras varios años con un hijo "aparentemente sano", con un diagnóstico que puede conllevar una pérdida de la ilusión y de las expectativas y un "proceso de duelo" o de "negación" (Hernández, Guerra y Aguilar 2012: 75). Teniendo en cuenta este problema de la objetividad, se ha evitado la opción 'a veces', a la que se puede recurrir tanto si un hito se consigue un 95\% de las ocasiones como si solo se logra un $2 \%$, y que además no ofrece información fiable a la hora de analizar la competencia pragmática de los participantes.

Para evaluar la correcta comprensión del cuestionario por parte de las familias, se realizó una prueba piloto con tres madres de niños con TEA que afirmaron comprender de forma clara los enunciados. No obstante, se realizaron algunos cambios: en primer lugar, en el apartado referente a la terapia, dado que la mayoría de familias habían probado no una, sino múltiples terapias con sus hijos, se optó por ofrecer la posibilidad de responder de forma extensa. Tras la prueba piloto también se añadieron algunas opciones intermedias -que en estos casos sí aportan información- en las preguntas en las que se vio necesario. Concretamente, se añadió 'depende de si es de su interés' a la pregunta relativa a prestar atención en una conversación; 'tiene dificultades para entonar de forma adecuada cuando está emocionado/a o enfadado/a', en el caso de la cuestión sobre entonación y 'ha aprendido las frases más habituales, pero no las puede entender si no se las explica alguien', en el apartado dedicado a revisar las dificultades a la hora de entender un significado no literal.

Por lo que respecta al rango etario, se optó por excluir a los menores de 6 años porque a esa edad todavía no han desarrollado algunos aspectos generales de la competencia comunicativa y porque muchos de los ítems a evaluar, como respetar el turno de palabra o la distancia interpersonal, no podían ser tenidos en cuenta. Además, con el fin de confirmar o refutar la hipótesis de la evolución y mejora de la competencia pragmática se analizaron las respuestas de forma global y también por edades, agrupando a las familias participantes en tres grupos, que se corresponden aproximadamente con la niñez mediana, la niñez intermedia y la adolescencia. Con esta división se pretendía observar si se producían cambios en la capacidad pragmática con el paso del tiempo y también conseguir una estadística más objetiva al evaluar los diferentes ítems de un individuo en comparación con los de su mismo grupo etario. Como se ha dicho, también preguntamos si habían recibido alguna terapia y cuál, para así recopilar la mayor información posible y discernir si este hecho podía tener alguna relación con los resultados. 
Desarrollo y mejora de la competencia pragmática en niños y adolescentes con trastorno del espectro autista y lenguaje funcional | L. Camargo y R. Cortés

\section{ANÁLISIS Y RESULTADOS}

De las 110 familias participantes, un $36,7 \%$ son padres y madres de niños y niñas de entre 6 y 8 años de edad, un 27,5\% de niños entre 9 y 12 y un 35,8\% de adolescentes a partir de 13 años. Un 0 '33\% de las preguntas no fueron contestadas por todos los participantes; en estos casos, los porcentajes se han efectuado en base a las respuestas dadas. En cuanto a las respuestas sobre la terapia, un $85,45 \%$, respondió que sus hijos habían recibido algún tipo de terapia, frente a un 14,55\% que no. En este caso, al tratarse de la única pregunta que no ofrece opciones sino una respuesta libre para especificar qué terapias se llevan a cabo, interpretamos como respuestas negativas tanto la ausencia de respuesta como el 'no'6.

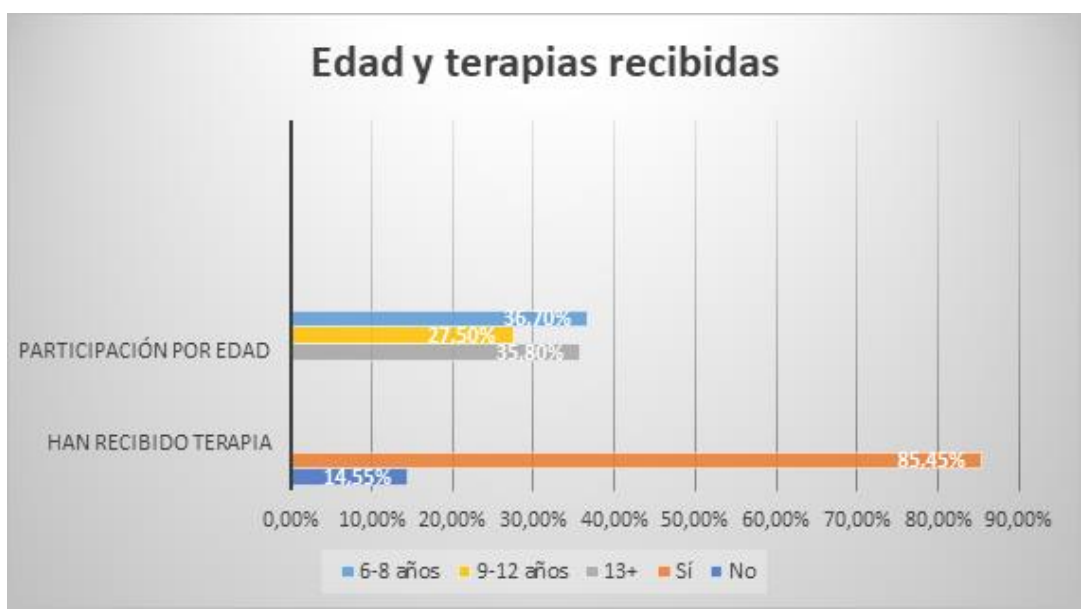

Gráfico 1. Edad y recepción de terapia

\subsection{La intención comunicativa}

Entre el grupo de preguntas dirigidas a evaluar la relación del hablante con el interlocutor, una de las respuestas más unánimes ha sido la relativa a la actitud de sus hijos cuando la conversación versa sobre uno de sus temas de interés: un 91,8\% de los encuestados afirmó que sus hijos hablan sin parar de sus intereses. En el análisis por edades los resultados de la primera y la segunda franja han sido muy similares; en el grupo adolescente, sin embargo, un 5,13\% de los encuestados -frente al $0 \%$ de los otros rangos, considera que sus hijos hablaban sin parar de sus temas de interés pero que ya no lo hacen.

Un $80 \%$ de los progenitores afirmó que sus hijos no perciben si se les escucha con atención o generan aburrimiento cuando hablan de sus intereses, cifra que asciende al $90 \%$ en el caso de

6 Analizamos de forma separada las respuestas de los padres cuyos hijos no realizan ninguna terapia y, aunque cabe reseñar que se trata de una muestra pequeña de 16 frente a los 110 participantes del análisis general, los resultados no se han incluido en el estudio al ser muy similares y no aportar información significativa. Las respuestas más marcadas por este grupo de progenitores han coincidido con las opciones preferidas en el cuestionario general a excepción de dos preguntas: las referentes a la distancia interpersonal y las estereotipias, las cuales han mostrado resultados más fragmentados en ambos casos. 
Desarrollo y mejora de la competencia pragmática en niños y adolescentes con trastorno del espectro autista y lenguaje funcional | L. Camargo y R. Cortés

los más pequeños. Solo un $7,27 \%$ considera que cambiarán de temática si son capaces de detectar signos de desinterés en la persona que les escucha y un 75,5\% afirmó que sus hijos cambian de temáticas de forma abrupta cuando hablan con otra persona. Teniendo en cuenta que la rigidez es uno de los rasgos de las personas con autismo, también preguntamos si consideraban que sus hijos tenían dificultades para aceptar otros puntos de vista durante una conversación. La respuesta más marcada fue claramente afirmativa (75,9\%), opción preferida en los tres grupos, si bien la franja del grupo de los adolescentes $(+13)$ ha vuelto a mostrar datos más positivos.

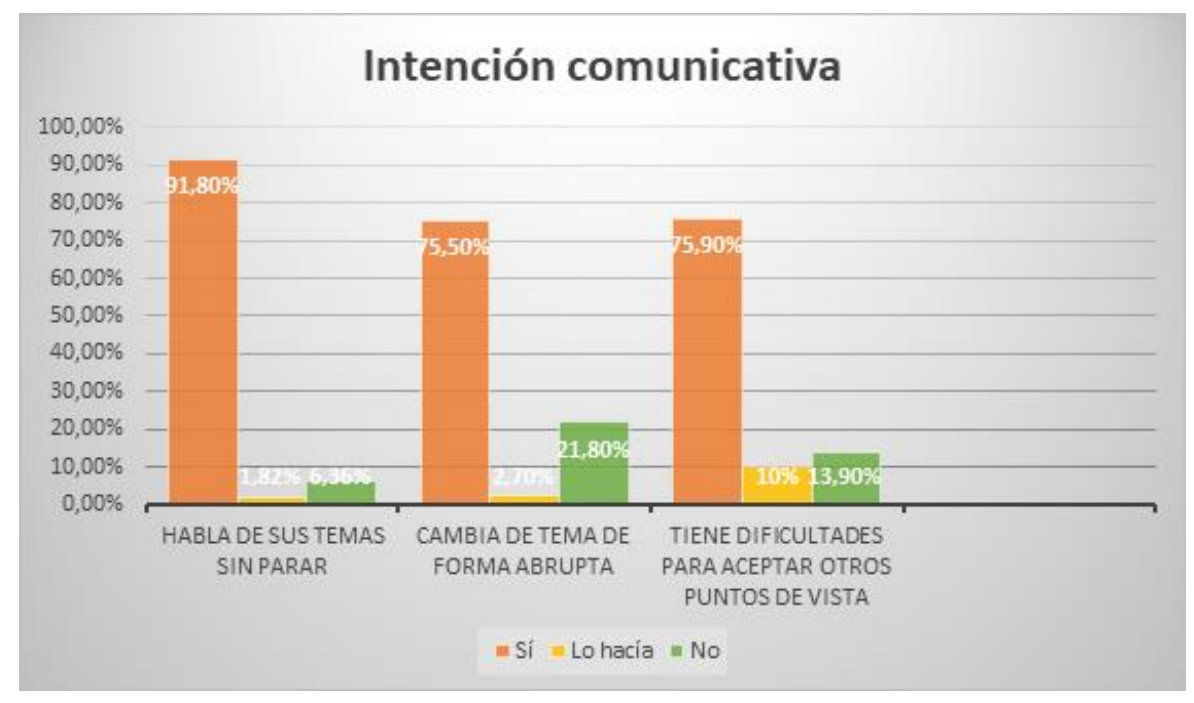

Gráfico 2. Intención comunicativa y relación con el interlocutor

Para evaluar si los participantes saben cambiar de registro en función de a quién se dirigen, se ha preguntado a los progenitores si su hijo trata de la misma manera a un profesor que a un niño y los resultados han mostrado que la mayoría (un 60\%) no establece diferencias. La opción 'no, lo hacía pero ha dejado de hacerlo' ha sido marcada por un $12,82 \%$ de los padres de adolescentes, un resultado que supone el doble del de la franja intermedia y más de cuatro veces el resultado del grupo de menor edad.

En esta misma línea, preguntamos por el uso de un vocabulario adecuado a la edad y al contexto, para incluir el uso contextualmente inadecuado de palabras impropias para la edad por su complejidad. El perfil lingüístico de la persona diagnosticada con SA se caracteriza precisamente por el uso de un vocabulario avanzado, que puede llegar a resultar rebuscado, pedante o demasiado culto. En este caso, las respuestas afirmativas suman un porcentaje más alto $(57,3 \%)$ que la negativa y en el análisis por edades, la percepción de los progenitores mejora con la edad: un 47,50\% de los padres del grupo 6-8 considera que sus hijos utilizan un 
Desarrollo y mejora de la competencia pragmática en niños y adolescentes con trastorno del espectro autista y lenguaje funcional | L. Camargo y R. Cortés

vocabulario adecuado, ya sea a tiempo o de forma tardía. En el grupo intermedio, estas cifras llegan al 56,67\% y en el adolescente al 66,67\%.

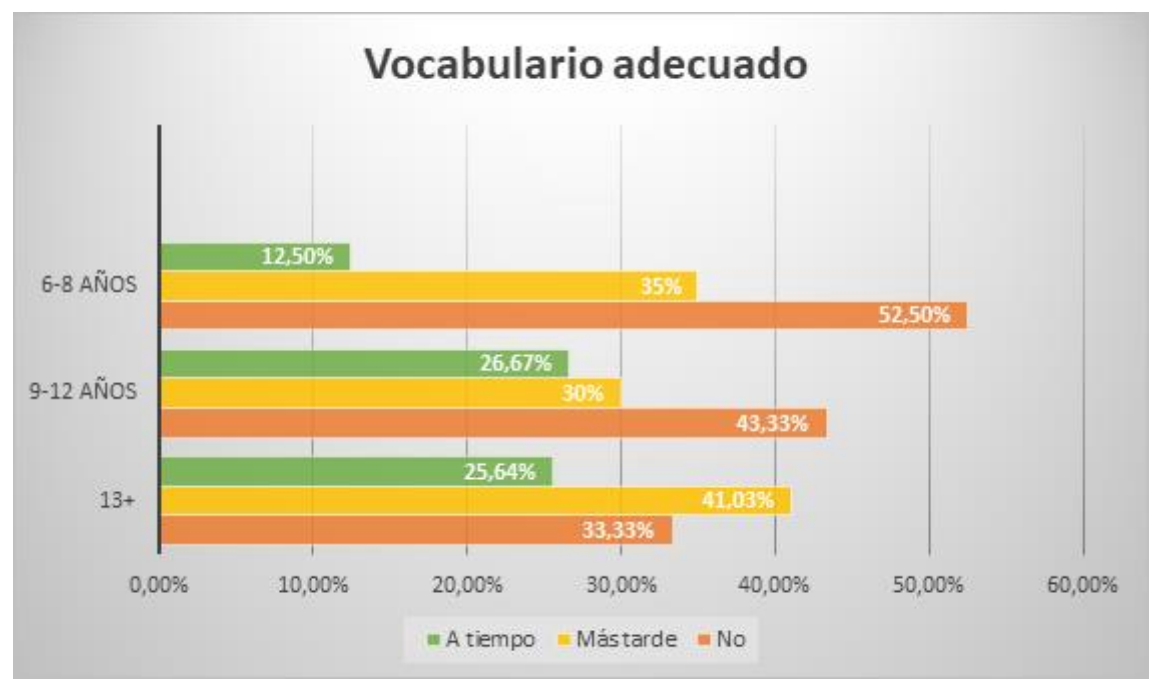

Gráfico 3. Vocabulario adecuado a la edad y al contexto

\subsection{La dinámica conversacional}

Las respuestas sobre habilidades conversacionales de los niños con autismo y lenguaje funcional de nuestra muestra revelan que estos no saben en qué momento intervenir en la conversación $(65,1 \%)$ y que casi un $60 \%$ no sabe respetar el turno de palabra $(58,2 \%)$; solo un $14,55 \%$ de los progenitores cree que, en caso de saber respetar el turno de palabra, seguirán haciéndolo si el tema de la conversación forma parte de sus intereses restringidos. Las dificultades en este terreno también quedan patentes al constatar que un $51,4 \%$ de los encuestados opina que sus hijos no pueden mantener una conversación fluida por teléfono. Aunque un $67 \%$ considera que niños y jóvenes sí responden a lo que se les pregunta durante una conversación, una inmensa mayoría (80\%) afirma que sus hijos prestarán atención en una conversación dependiendo del interés que esta les suscite.

En el análisis por franjas de edad cabe reseñar que son los más pequeños los que muestran más dificultades para saber cuándo intervenir en una conversación: un $76,92 \%$ no sabe cuándo hacerlo, cifra similar a la del grupo de 9-12 años (73,33\%), que sin embargo desciende casi 30 puntos $(46,15 \%)$ en el caso de los adolescentes. En la pregunta referente al respeto del turno de palabra, los padres de adolescentes han optado preferentemente por un aprendizaje tardío $(53,85 \%)$ frente a los otros dos grupos, que en su mayoría niegan la adquisición de esta habilidad; en el caso de que el tema de conversación sea de gran interés para el niño, los tres 
Desarrollo y mejora de la competencia pragmática en niños y adolescentes con trastorno del espectro autista y lenguaje funcional | L. Camargo y R. Cortés

grupos coinciden en que no respetará el turno, pero los datos son más positivos en el grupo +13 que en los otros dos.

No hay grandes diferencias por edad en la cuestión referente a la atención en una conversación, mientras que en el apartado que tiene como objeto comprobar si los niños y jóvenes responden a lo que se les pregunta, en los tres grupos se ha elegido un mayor número de veces la opción marcada en los resultados generales: un aprendizaje tardío.

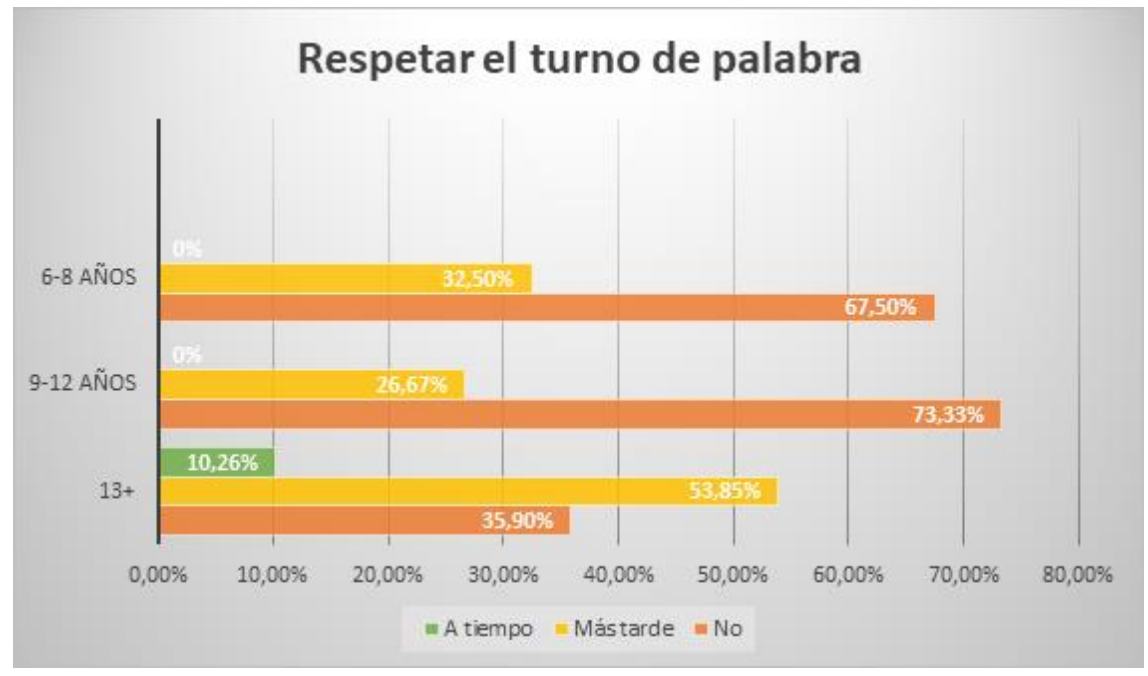

Gráfico 4. Turno de palabra

\subsection{La comunicación no verbal}

Entre el grupo de preguntas destinadas a evaluar la capacidad de interpretación de las señales no verbales de los niños y jóvenes, la referente al contacto visual ha mostrado que la mayoría de los encuestados $(64,2 \%)$ considera que sus hijos o hijas miran a la persona que les habla, aunque tardaron más en adquirir el aprendizaje que sus iguales; con todo, aunque un $46,4 \%$ afirma que sus hijos han aprendido a mantener la mirada -también de forma tardía-, un 39,1\% opina que aún no han adquirido este aprendizaje. El contacto ocular también se ve afectado si la conversación se produce con una persona desconocida o en un nuevo ambiente; en ese caso, solo un $22,94 \%$ de los progenitores encuestados considera que su hijo o hija mantendrá la mirada.

Un $67,3 \%$ de los padres, por otra parte, indicó que sus hijos han aprendido a reconocer los gestos más frecuentes pero que ante interlocutores desconocidos o en ambientes diferentes les costará entenderlos $(58,18 \%)$ o directamente no los entenderán (21,82\%). En el análisis por edades, llama la atención que a pesar de la coincidencia en que sus hijos o hijas muestran dificultades, son los más pequeños los que reciben una mejor puntuación: un $30 \%$ de los 
Desarrollo y mejora de la competencia pragmática en niños y adolescentes con trastorno del espectro autista y lenguaje funcional | L. Camargo y R. Cortés

progenitores asegura que sí entenderán los gestos, frente al 20,51\% de los adolescentes y solo el 6,67\% de la franja intermedia. En el formulario también se ha tratado de averiguar si durante una conversación los hijos e hijas de los participantes presentan estereotipias motoras 7 . En este caso, se han ofrecido tres respuestas: afirmativa, negativa y también la opción de que se trate de una conducta extinguida. Los resultados han sido más ajustados: un $40 \%$ ha afirmado que sus hijos realizan estereotipias mientras hablan, un $36,4 \%$ que no las hacen y el 23,6\% que las hacían antes pero ya no. En el análisis por edades, los grupos de menor edad han seleccionado en su mayoría la respuesta 'sí, mientras que en el rango adolescente la opción más marcada ha sido la que alude a una conducta extinguida.

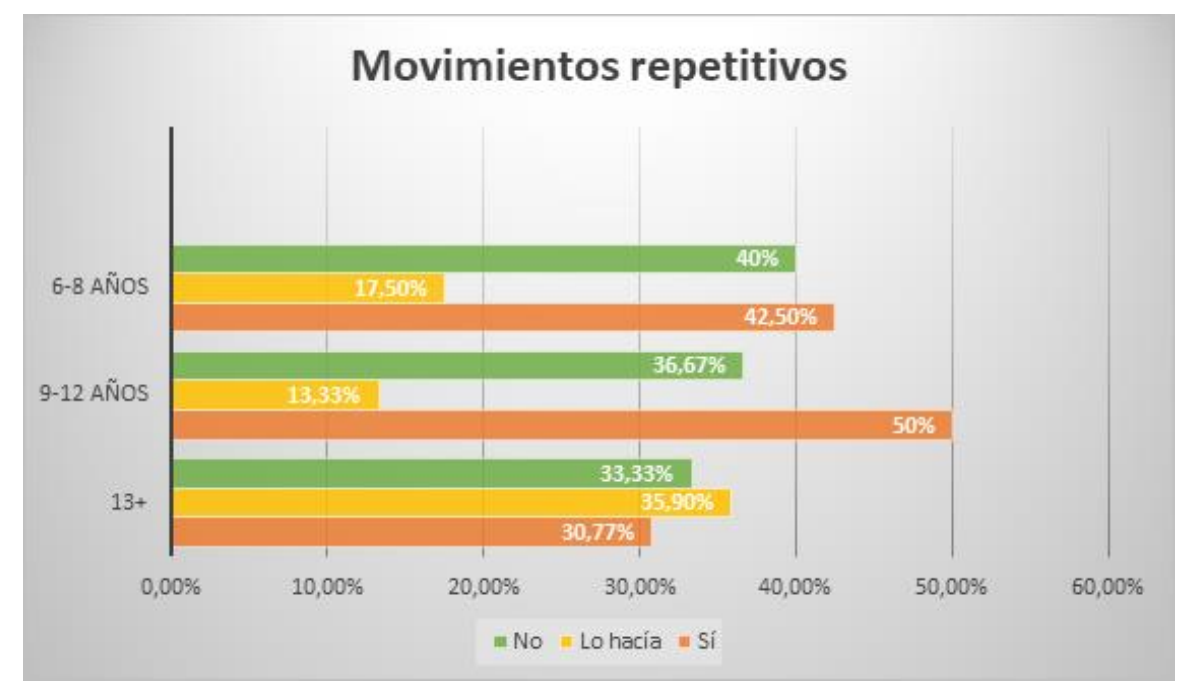

Gráfico 5. Estereotipias motoras durante la conversación

En el terreno de la proxemia, un $44,5 \%$ de los encuestados afirma que sus hijos han aprendido a mantener la distancia, aunque más tarde que sus iguales. Por edades, las franjas 9-12 y 13+ han coincidido en la opción más marcada, la del aprendizaje tardío, mientras que en el caso de los más pequeños la respuesta más votada ha sido el 'no', que han pulsado el 50\% de padres.

7 Movimientos "repetitivos, rítmicos y carentes de propositividad" que pueden manifestar personas con TEA (Eirís-Puñal 2014: 81). 


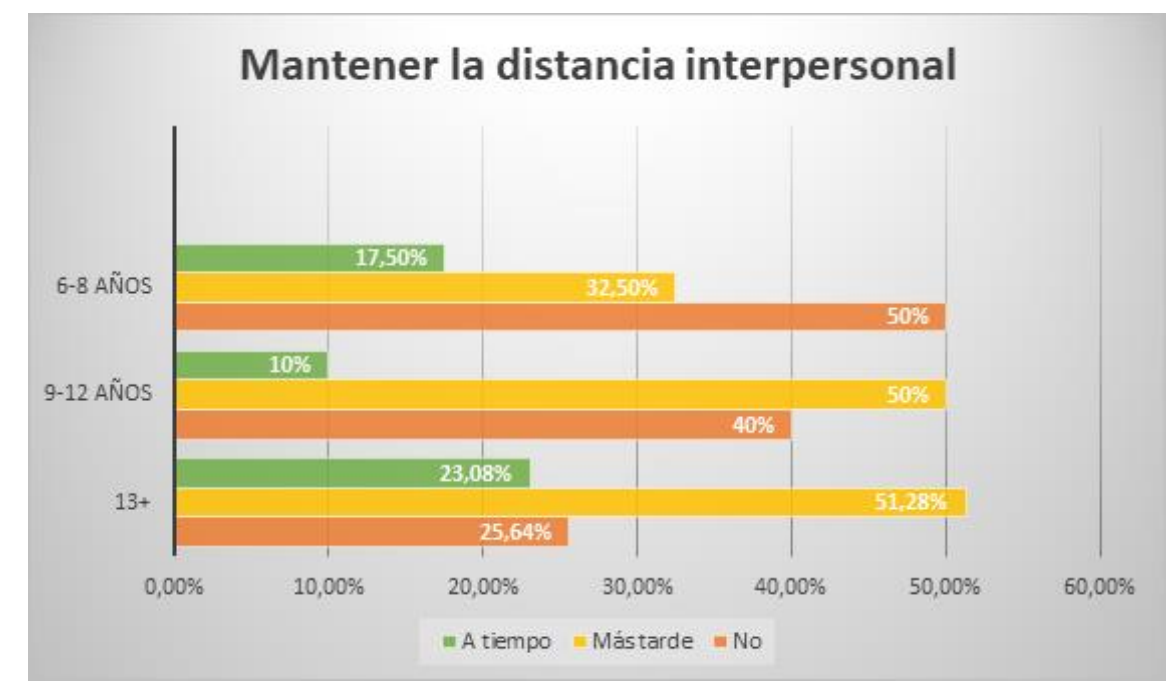

Gráfico 6. Aspectos proxémicos

Otro de los aspectos paralingüísticos que se puede ver afectado en estos perfiles es la prosodia. En la pregunta sobre si sus hijos emplean una entonación adecuada al hablar ofrecimos además de las opciones negativa y la de la adquisición, a tiempo o tardía, del aprendizaje, las siguientes respuestas: 'Tiene dificultades para entonar de forma adecuada cuando está emocionado/a o enfadado/a', dado que no es infrecuente que tengan problemas a la hora de autorregular estas emociones, y 'Habla (o hablaba) en un mismo tono)', pues algunos niños y jóvenes con TEA pueden presentar una entonación monótona.

La respuesta seleccionada un mayor número de veces ha sido la referente a las dificultades para entonar por emoción o enfado, que ha marcado un 57,8\% de los encuestados. En segundo lugar, a mucha distancia, se encuentra el aprendizaje tardío $(13,8 \%)$ y en tercero, con un $11,9 \%$, el aprendizaje a tiempo. Los porcentajes han sido bastante similares en las tres franjas de edad. 


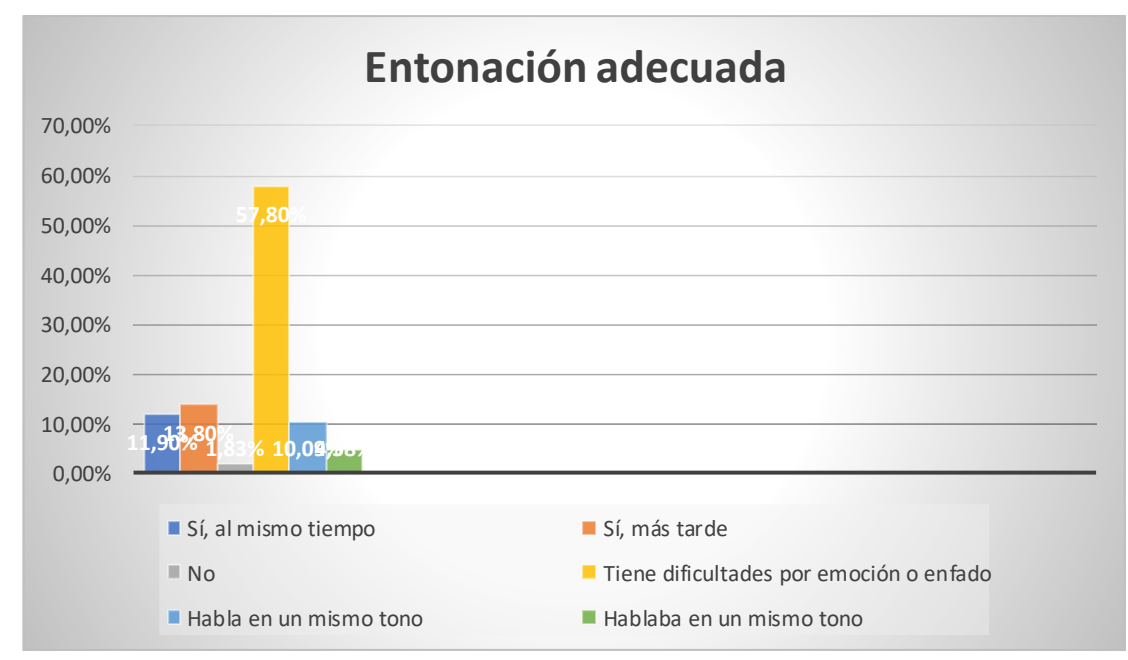

Gráfico 7. Patrones de entonación

\subsection{La comprensión del lenguaje figurado y las unidades fraseológicas}

A partir de dos ejemplos de UFS (¿entiende frases del tipo "ponerse las pilas" o "estar en las nubes"?, preguntamos por la capacidad para interpretar estas unidades de significado no composicional. Añadimos una nueva opción a la negativa y al aprendizaje adquirido, ya fuera este a tiempo o tardíamente: haber aprendido las frases hechas más habituales, pero no ser capaces de entenderlas sin ayuda. Los resultados, de hecho, muestran que la nueva opción ha sido la más votada por los progenitores, concretamente por un $65,5 \%$. Por edades, son los padres de los más pequeños los que niegan la capacidad de entender este tipo de construcciones con mayor contundencia: un 35\% considera que sus hijos no pueden entenderlas frente al $20 \%$ de los padres del grupo $9-12$ y solo un $7,69 \%$ de las familias de los adolescentes. 


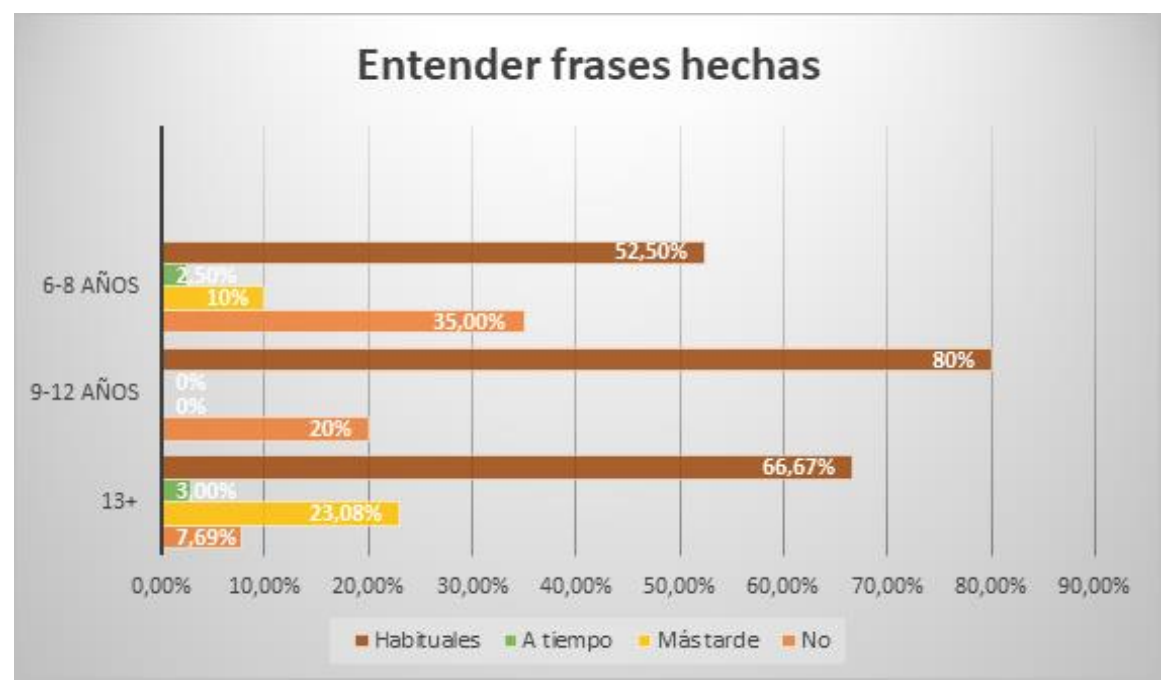

Gráfico 8. Comprensión del lenguaje figurado

Ante las dificultades para entender dobles sentidos, ironías y bromas se ha optado por preguntar a los participantes en el cuestionario si consideran que, en el caso de que sus hijos o hijas entiendan las bromas, comprenden su contenido si no las han escuchado con anterioridad. Habilitamos la opción 'no las entiende' para quienes creen que este aprendizaje no se ha adquirido. Un $47,3 \%$ se ha decantado por la respuesta intermedia 'depende de si está en un ambiente de confianza' y solo un 3,6\% de los participantes afirma que sus hijos podrían distinguir una broma que no han escuchado nunca.

\subsection{Hipersensibilidad sensorial}

Un alto porcentaje de los encuestados $(91,8 \%)$ ha considerado que los estímulos externos pueden condicionar las respuestas de sus hijos en una conversación; los más pequeños son los que se ven más afectados por la sobrecarga sensorial.

\section{CONCLUSIONES}

Los resultados muestran que los niños y jóvenes de las familias participantes presentan numerosas dificultades para lograr una comunicación exitosa, dado que para ello es necesario saber escuchar, respetar el turno de palabra, mantenerlo y cederlo, habilidades sencillas para una persona neurotípica que son de gran complejidad para una persona con autismo, incluso cuando existe lenguaje funcional. Como hemos podido comprobar, estas personas tienden, además, a seleccionar temas de conversación centrados en sus propios intereses, sin tener en cuenta los del interlocutor ni su conocimiento respecto al tema de conversación. A este último hecho hay que sumar las dificultades de los niños y jóvenes con Asperger o TEA grado 1 para comprender el sentido figurado y las señales no verbales, sin olvidar la influencia que puede tener una sobrecarga sensorial en su atención y respuestas. 
Desarrollo y mejora de la competencia pragmática en niños y adolescentes con trastorno del espectro autista y lenguaje funcional | L. Camargo y R. Cortés

Estos resultados coinciden con el perfil lingüístico descrito a la vez que aportan más información respecto a nuestra hipótesis inicial: determinados aprendizajes relativos a la competencia pragmática se acaban adquiriendo, aunque sea de forma tardía. Entre los más afianzados figuran responder a lo que se pregunta, mirar al interlocutor y mantener la mirada, mantener la distancia interpersonal y el uso de un vocabulario adecuado. Las puntuaciones en la mayoría de los ítems del cuestionario son más positivas a mayor desarrollo evolutivo, lo que demuestra que la competencia pragmática mejora a medida que el niño crece, se socializa y aprende a comunicarse con los demás. Los datos recogidos, de hecho, muestran que los adolescentes $(+13)$ obtienen porcentajes más elevados en el apartado de aprendizajes adquiridos que los participantes de menor edad (6-12 años) en casi todas las áreas analizadas.

Creemos que la diferencia en cuanto a mejora de la competencia pragmática entre los otros dos grupos etarios (6-8 y 9-12 años) no es tan evidente (muestran datos similares en muchas ocasiones o diferencias más discretas) debido a la dificultad de diagnóstico, lo cual podría implicar una mayor dificultad por parte de las familias a la hora de distinguir características propias del trastorno: ${ }^{8}$ Por otra parte, determinadas conductas comunicativas -intereses restringidos, problemas de atención y a la hora de respetar turnos de palabra, distancia interpersonal, etc.- no resultan tan llamativas en el primer grupo de edad, lo que podría explicar que la franja intermedia, con edades en las que las diferencias con el grupo de niños neurotípicos ya son más evidentes, llegue incluso a obtener peores puntuaciones en algunos apartados. Es lo que sucede con el hecho comentado en el apartado de análisis relativo al reconocimiento de los gestos más frecuentes ante interlocutores desconocidos o en ambientes diferentes.

Los resultados reflejan asimismo que hay ciertos aspectos de la dimensión social del lenguaje que son muy difíciles de adquirir -la mayoría de los participantes, de hecho, no lo han conseguido- como son todos los relacionados con la intención comunicativa y la capacidad de percibir la intención y necesidades del interlocutor para conseguir una comunicación bidireccional. El análisis de las respuestas recabadas también pone de manifiesto que una parte del aprendizaje es únicamente memorística (gestos, frases hechas), por lo que la respuesta puede no ser la adecuada en nuevos contextos. La dificultad de las personas con TEA para generalizar aprendizajes se demuestra también en el hecho de que pese a haber adquirido determinadas habilidades son muy pocos los que podrán ponerlas en práctica con interlocutores desconocidos o en nuevos escenarios.

Por último, para futuras investigaciones, consideramos la posibilidad de abordar la competencia pragmática de niños y jóvenes con autismo y lenguaje funcional desde la perspectiva de género. Esta propuesta cobra especial importancia si tenemos en cuenta que, como han afirmado diversos autores, las niñas con este diagnóstico "se encuentran mejor

8 Ciertos problemas se confunden con las propias características del niño o se achacan al desarrollo, por lo que muchos casos no se detectan hasta el inicio de la escolarización o incluso con posterioridad (Freire et al. 2007: 41).

Normas (ISSN: 2174-7245)| 
Desarrollo y mejora de la competencia pragmática en niños y adolescentes con trastorno del espectro autista y lenguaje funcional | L. Camargo y R. Cortés

equipadas para la comprensión social y cuentan con más habilidades socio-comunicativas en comparación con los niños con TEA” (García López 2019).

\section{BIBLIOGRAFÍA}

American Psychiatric Association (2013): Diagnostic and statistical manual of mental disorders (5a edición), (DSM5), Washington DC.

Artigas, Josep (2000): «Aspectos neurocognitivos del síndrome de Asperger», Revista de Neurología Clínica, 1, 34-44.

Baron-Cohen, Simon (2001): «Theory of mind autism: A review», International Review of Research in Mental Retardation: Autism, 23, 169 -184 .

Barthélémy, Catherine et alii (2019): Personas con trastorno del espectro del autismo. Identificación, comprensión, intervención, Autismo-Europa [en línea]: https://www.autismeurope.org/wpcontent/uploads/2019/11/People-with-AutismSpectrum-Disorder.-Identification-

Understanding-Intervention_Spanishversion.pdf [Consulta: 29/11/2020].

Camargo Fernández, Laura (2009): «La metapragmática» en Ruiz Gurillo, Leonor y Xosé Padilla, eds., Dime cómo ironizas y te diré quién eres. Una aproximación pragmática a la ironía, Frankfurt, Peter Lang, 89-107.

Camargo Fernández, Laura y Beatriz Méndez Guerrero (2014): «La pragmática del silencio en la conversación en español. Propuesta taxonómica a partir de conversaciones coloquiales», Sintagma, 26, 103-118, https://www.raco.cat/index.php/Sintagma/articl e/view/293006/381455.

Cestero Mancera, Ana Ma (2000): El intercambio de turnos de habla en la conversación, Alcalá de Henares, Servicio de Publicaciones de la Universidad de Alcalá.

Cestero Mancera, Ana Ma (2014): «Comunicación no verbal y comunicación eficaz», ELUA: Estudios de Lingüística. Universidad de Alicante, 28, 125150, https://doi:10.14198/ELUA2014.28.05.

Confederación Asperger España (s. a.): «El síndrome de Asperger: qué es y características principales», [en línea]: $<$ https://www.asperger.es/que_es_asperger.html $>$ [Consulta: 15/10/2020].
Cortés García, Regina (2020): Autismo con lenguaje funcional: un enfoque pragmático (trabajo de fin de grado), Universitat de les Illes Balears: http://hdl.handle.net/11201/153808.

Eirís-Puñal, Jesús (2014): «Trastornos motores en los trastornos del neurodesarrollo. Tics y estereotipias», Revista de Neurología, 58 (Suplemento 1), 77-82.

Flavell, John H. (2004): «Theory-of-mind development: Retrospect and prospect», MerrillPalmer Quarterly, 50, 274-290, https://doi.org/10.1353/mpq.2004.0018.

Freire Prudencio, Sandra et alii (2007): Un acercamiento al síndrome de Asperger: una guía teórica y práctica, Sevilla, Asociación Asperger Andalucía.

Gallardo Paúls, Beatriz (1996): Análisis conversacional y pragmática del receptor, Valencia, Episteme.

García López, Cristina (2019): «Las reinas del camuflaje: niñas con Trastorno del Espectro del Autismo (TEA)», Faros, Hospital Sant Joan de Déu, Barcelona, [en línea]: $<$ https://faros.hsjdbcn.org/es/articulo/reinascamuflaje-ninas-trastorno-espectro-autismotea> [Consulta: 20/11/2020].

Happé, Francesca (1999): «Autism: Cognitive deficit or cognitive style?», Trends in Cognitive Sciences, 3, 216-222.

Hernández Martínez, Violeta Adlai, Berenice Calixto Guerra e Ileana Elvira Aguilar Castañón (2012): «Aspectos psicológicos de familiares de personas diagnosticadas con Trastorno del Espectro Autista (TEA)», Revista Intercontinental de Psicología y Educación, 14 (1), 73-90.

Kalandadze, Tamar, Valentina Bambini y Kari-Anne B. Næss (2019): A systematic review and metaanalysis of studies on metaphor comprehension in individuals with autism spectrum disorder: Do task properties matter?, Applied Psycholinguistics, 40 (6), 1421-1454, https://doi.org/10.1017/S0142716419000328.

Maristany, Marta (2002): «El alumno con Síndrome de Asperger en la Escuela Primaria», Servicio de 
Desarrollo y mejora de la competencia pragmática en niños y adolescentes con trastorno del espectro autista y lenguaje funcional | L. Camargo y R. Cortés

Neurología del Hospital Sant Joan de Déu, Barcelona, [en línea]: $<$ http://www.jmunozy.org/files/NEE/asperger/IJ ORNADAS_ASPERGER_Y_EDUCACION/S.\% 20Asperger\%20y\%20EDUCACION/El\%20alum no\%20SA\%20en\%20la\%20Escuela\%20Primaria .M.Maristany.pdf> [Consulta: 12/09/2020].

Martín-Borreguero, Pilar (2005): «Perfil lingüístico del individuo con síndrome de Asperger: implicaciones para la investigación y la práctica clínica», Revista de Neurología, 41 (Suplemento $1)$,

115-122,

http://www.sld.cu/galerias/pdf/sitios/rehabilitac ion-logo/perfil linguistico de asperger.pdf.

Martos Pérez, Juan (2007): «¿Cómo y qué medida (sic) el lenguaje de las personas con síndrome de Asperger puede ser discapacitante?», Síndrome de Asperger: aspectos discapacitantes $y$ valoración, Dossier encargado por la Federación Asperger España, 7-14, https://www.aspergeraragon.org.es/wordpress/ wp-content/uploads/2017/01/DOSSIERASPERGER-Valoracion-Discapacidad.pdf.

OMS (2020): Clasificación internacional de enfermedades 10.a revisión, modificación clínica, edición española, Edición electrónica de la CIE-10-ES Diagnósticos [en línea]: <eciemaps.mscbs.gob.es/ecieMaps/browser/inde x_10_mc.html> [Consulta: 07/11/2020].

Parsons, Lauren et alii (2017): «A systematic review of pragmatic language interventions for children with autism spectrum disorder», PLOS ONE 12 (4),

https://doi.org/10.1371/journal.pone.0172242.

Pastor-Cerezuela, Gemma, Juan Carlos Tordera Yllescas, Francisco González-Sala, Maite Montagut-Asunción, María-Inmaculada Fernández-Andrés (2018): "Comprehension of generalized conversational implicatures by children with and without autism spectrum disorder», Frontiers in Psychology, 9, 272, https://doi.org/10.3389/fpsyg.2018.00272.

Pexman, Penny M. et alii (2011): «Processing of Ironic Language in Children with HighFunctioning Autism Spectrum Disorder», Journal of Autism and Developmental Disorders,
41: 1097-1112, https://10.1007/s10803-0101131-7.

Reboul, Anne, Sabine Manificat y Nadège Foudon (2012): «Autism from a cognitive-pragmatic perspective», en Schmid, Hans-Jörg, ed., Cognitive Pragmatics, Berlin, De Gruyter Mouton, 317-344.

Rodríguez Muñoz, Francisco José (2012): «La conciencia pragmática de adultos con síndrome de Asperger», Revista de Logopedia, Foniatría y Audiología, 32, 21-31, https://doi:10.1016/j.rlfa.2012.01.002.

Rodríguez Muñoz, Francisco José (2013): Evaluación pragmática de niños con síndrome de Asperger, Munich, Lincom.

Rodríguez Muñoz, Francisco José (2019): «The construction of cooperative and inferential meaning by children with Asperger syndrome», Topics in Linguistics, 20 (1), 54-67, https://doi.org/10.2478/topling-2019-0004.

Searle, John R. (1993): «Metaphor», en Ortony, Andrew, ed., Metaphor and Thought, Cambridge, Cambridge University Press, 83111.

Schaeken, Walter, Marie Van Haeren y Valeria Bambini (2018): «The understanding of scalar implicatures in children with autism spectrum disorder: dichotomized responses to violations of informativeness", Frontiers in Psychology, 9, 1266, https://doi.org/10.3389/fpsyg.2018.01266.

Timofeeva, Larissa y Leonor Ruiz Gurillo (2018): «Fraseología y humor: de semántica y pragmática», en Pamies, Antonio, Isabel María Balsas y Alexandra Magdalena, eds., Lenguaje figurado y competencia interlingüística (I), Granada, Comares, 151-163.

Tordera Yllescas, Juan Carlos (2007): «Trastorno de espectro autista: delimitación lingüística», ELUA: Estudios de Lingüística. Universidad de Alicante, 21, 1-15, http://dx.doi.org/10.14198/ELUA2007.21.15.

Twachtman-Cullen, Diane (1998): «Language and Communication in high-functioning autism and Asperger syndrome», en Schopler, Eric, Gary B. Mesibov y Linda J. Kunce eds., Asperger syndrome or high-functioning autism?, New York, Plenum Press, 199-223. 
Desarrollo y mejora de la competencia pragmática en niños y adolescentes con trastorno del espectro autista y lenguaje funcional | L. Camargo y R. Cortés 